\title{
Clinical Research: The Future of the Molecular Imaging Technologist — A White Paper Presented by Members of the Graduate Stakeholders Committee of the SNMMI Technologist Section
}

Scott Holbrook ${ }^{1}$, John Baldwin ${ }^{2}$, C. David Gilmore ${ }^{3}$, Robert Henkin ${ }^{4}$, William Hubble ${ }^{5}$, Christina Kiss ${ }^{6}$, Angela Macci Bires ${ }^{7}$, April Mann ${ }^{8}$, Kasey P. Nelson ${ }^{9}$, Mary Anne Owen ${ }^{10}$, Katie Sobey ${ }^{11}$, Alan Stuckey ${ }^{12}$, Nikki Wenzel-Lamb ${ }^{6}$, Debbie Wilkinson ${ }^{13}$, and Nancy A. Wintering ${ }^{14}$

${ }^{1}$ Invivo Molecular Imaging LLC, Gray, Tennessee; ${ }^{2}$ Division of Nuclear Medicine, University of Alabama at Birmingham, Birmingham, Alabama; ${ }^{3}$ Regis College, Weston, Massachusetts; ${ }^{4}$ UNM Ltd., Chicago, Illinois; ${ }^{5}$ Saint Louis University, St. Louis, Missouri; ${ }^{6}$ Society of Nuclear Medicine and Molecular Imaging, Reston, Virginia; ${ }^{7}$ Robert Morris University, Pittsburgh, Pennsylvania; ${ }^{8}$ Hartford Hospital, Hartford, Connecticut; ${ }^{9}$ Clinical Imaging Physics Group, Duke University, Durham, North Carolina; ${ }^{10}$ Georgia Regents University, Augusta, Georgia; ${ }^{11}$ Bracco, Princeton, New Jersey; ${ }^{12}$ Graduate School of Medicine, University of Tennessee, Knoxville, Tennessee; ${ }^{13}$ Wheeling Jesuit University, Wheeling, West Virginia; and ${ }^{14}$ Thomas Jefferson University, Philadelphia, Pennsylvania

$\mathbf{T}$ he passage of the Food and Drug Administration Modernization Act of 1996 set into motion the eventual pathway for approval of biomarkers used in PET. This was the sentinel event in the history of molecular imaging as well as the lives and careers of countless patients and molecular imaging professionals. Subsequent federal regulations and guidance documents have established the environment for approval of future drugs. The translation of a concept or theory from the abstract to the clinical environment, or from the bench to the patient bedside, is a multidisciplinary effort often involving large teams of scientists and clinical professionals. The translation of novel biomarkers for use as companion diagnostic agents or research tools in nuclear medicine and molecular imaging is an evolving field. Because of the complexity of the imaging agents, the requirement for local drug manufacturing due to a short radioactive half-life of the agents, technical issues associated with accurate data collection, and patient management considerations, specifically certified nuclear medicine technologists are well suited to participate in research and development activities associated with these drugs.

Opportunities for molecular imaging professionals to participate in research and development have been the focus of numerous professional societies including the Society of Nuclear Medicine and Molecular Imaging

Received Jul. 29, 2014; accepted Jul. 29, 2014.

For correspondence or reprints contact: Scott Holbrook, 101 Dillon Ct. Gray, TN 37615.

E-mail: sholbrookcps@comcast.net

Published online Aug. 14, 2014.

COPYRIGHT (C) 2014 by the Society of Nuclear Medicine and Molecular Imaging, Inc.

DOI: 10.2967/jnmt.114.146373
(SNMMI). In particular, SNMMI's Center for Molecular Imaging Innovation and Translation convened a task force to focus on development of a curriculum for molecular imaging scientists who are involved with the translation of molecular imaging agents. This task force identified the domains of expertise necessary to successfully translate a drug or technology. These domains included the regulatory, preclinical trial phase during which a clinical dilemma is identified, identification of the target and selection of potential biomarkers for characterization of the target, preclinical imaging to validate the target biomarker and biodistribution, interfacing with medical devices, early-phase clinical development, preparation of the clinical package before initiation of human studies, clinical trial design and implementation, and clinical manufacturing postapproval activities.

\section{BACKGROUND}

The SNMMI Technologist Section has spent many years researching and creating a mid-level provider of nuclear medicine services and developing a curriculum that would coincide with this new advanced-level position. Now, several years later, the SNMMI Technologist Section understands the importance of creating additional master's-level programs that will provide advanced educational opportunities to members. During the graduate stakeholders meeting this past December, the task force members developed a statement that was approved by the SNMMI board of directors during the 2014 mid-winter meeting. The statement outlines the Society's position on graduate-level education and the Society's stake in future training programs.

SNMMI supports advanced associate degrees and graduate education for nuclear medicine and molecular imaging 
technologists. Although the SNMMI continues to support the master of business administration, master of public health, and other established master's degree programs, there is an important special interest in the continued growth of graduate programs in molecular clinical research.

To that end, it is understood by the community that nuclear medicine and molecular imaging are unique among other medical imaging specialties in that the modalities rely not only on imaging techniques but also on organ- or pathology-specific biomarkers that, together, provide the answer to important clinical questions. Additionally, nuclear medicine and molecular imaging professionals must also possess expertise in targeted radionuclide therapies that are gaining acceptance as routine clinical applications for treatment of various diseases. These characteristics make nuclear medicine and molecular imaging a powerful profession that has tremendous potential. This has led to some of the most significant challenges within the field, which must be overcome in order to ensure that the potential of nuclear medicine and molecular imaging will be realized.

The need to provide reproducibility, quality, and safety for patients in a cost-effective manner has never been greater. It is the intersection of these elements that defines the value offered by nuclear medicine and molecular imaging procedures. To sacrifice any one point devalues the end result to the patient and clinician. In a time when each health-care dollar spent is closely examined, we must understand the importance of how we deliver this value.

A 2007 study (1) reported that although much of the technologist shortage occurred with the swift implementation of PET/CT, the shortage may once again occur with the introduction of PET/MR and "an increasing reliance on small-animal radiotracer imaging in drug discovery and research in academic medical centers and in the biotechnology and pharmaceutical industry."

Clinical research technologists and other imaging scientists are key personnel who contribute substantially to the success of an imaging clinical trial. Technologists are responsible for obtaining high-quality images, collecting numerous other data points, and documenting it all; however, few receive adequate training in clinical research practices, which are different from clinical practice standards. Without well-trained clinical research technologists and imaging scientists, a study may fail to produce measurable outcomes. Millions of dollars and years of research can be wasted, not to mention the loss of a potentially successful drug development or treatment regimen for patients.

In an editorial (2) by the graduate stakeholders chair, C. David Gilmore, it was reported that "there are also other major areas of growth, with multiple jobs, that we should focus on-all of which build on the knowledge and skills acquired in a nuclear medicine program....research, or more specifically, the clinical research associate. Many biotech organizations, pharmaceutical companies, universities, and academic medical centers have positions for clinical research associates. These individuals ensure the compli- ance of research studies, act as liaisons between groups, implement policies and procedures, and maintain the federal, state, and local files required for research projects. Clinical research associates must be knowledgeable about medical and clinical terminology, be able to complete drug reconciliations and verify protocols, and have the communication and interpersonal skills needed for teamwork and skilled patient care."

\section{PROPOSED EDUCATIONAL DOMAINS}

The nuclear medicine technologist is a highly specialized health-care professional who works closely with the nuclear medicine physician. Some of the technologist's primary responsibilities are to prepare and administer radioactive chemical compounds, known as radiopharmaceuticals; perform patient imaging procedures using sophisticated radiationdetecting instrumentation; accomplish computer processing and image enhancement, analyze biologic specimens in the laboratory; and provide images, data analysis, and patient information to the physician for diagnostic interpretation.

The proposed clinical research curriculum assumes that students will have a significant understanding of nuclear medicine and molecular imaging and have graduated from an accredited program with a bachelor's degree in nuclear medicine, biology, chemistry, or a related field. Some related postgraduate work experience is expected. In addition, it is recommended that this individual be a certified nuclear medicine technologist who is seeking to advance into clinical research. This transition from a clinical setting to clinical research will be in the form of a graduate-level program consisting of the domains described in the next sections.

\section{Introduction to Research}

The most important concept in moving from a clinical setting to a research setting is to understand the difference between clinical practice and clinical research. Clinical practice is the established (validated) treatments and diagnostic tests that are used for medical decision making and patient care, whereas clinical research is evidence that is collected to establish (validate) treatment or diagnostic tests in order to guide future medical decision making and patient care. Clinical research helps determine the safety and effectiveness of medications, devices, diagnostic products, and treatment regimens intended for human use. These may be used for prevention, treatment, diagnosis, or relief of symptoms of a disease. Once an unmet clinical need has been identified and the appropriate clinical question has been determined, the researcher must be familiar with the broad framework of clinical research infrastructure in order to advance a theory toward translation. Areas that must be addressed in this domain include, but are not limited to, identifying an unmet clinical need, understanding and ensuring necessary regulatory filings, identifying intellectual property issues, identifying sources of funding and postapproval reimbursement challenges, and overseeing translation and commercialization. 


\section{Regulatory and Quality Assurance}

Quality assurance activities consist of oversight to ensure that the identity, strength, quality, and purity of a given drug product are consistent with labeling. Specifically, the quality assurance activities of a given institution routinely include reviewing and accepting or rejecting proposed changes to procedures or quality specifications; examining labeling, packaging, and dosage forms to ensure consistency with specifications; overseeing and conducting investigations requiring corrective and preventative action to ensure that the root cause of a deviation is identified and, if necessary, measures are taken to prevent the same deviation from occurring again; routinely reviewing batch records to ensure completeness and accuracy; conducting batch trend analysis to assess manufacturing consistency; and performing periodic audits associated with manufacturing.

Molecular imaging and nuclear medicine professionals who participate in a quality unit or in regulatory affairs must be intimately familiar with good manufacturing processes (GMPs), good laboratory practices, a given institution's standard operating procedures, drug manufacturing best practices, and numerous regulations. Some of these important regulations are 21 Code of Federal Regulations (CFR) part 212, 21 CFR part 211, U.S. Pharmacopeia (USP) 823, and USP 797.

\section{Preclinical Techniques}

In a research setting, such as a preclinical imaging facility, the molecular imaging and nuclear medicine specialist's role will become dynamic relative to the availability of imaging platforms in the facility, the demands of the specific research project, and the animal models under study. Knowledge of radioisotopes, radiopharmaceuticals, radiation safety, anesthesia, the biology of the subject, and the biochemistry of the radiotracer becomes important. Much of the work in the preclinical setting uses novel radiotracers that are in development; therefore, protocols often evolve. A broad understanding of numerous imaging modalities and a firm grasp of the biologic impact as a novel radiotracer moves from in vitro discovery to in vivo validation and efficacy studies are of critical import.

Because of the complexity of developing new drugs for human use, technologists play a critical role by leveraging their training and practical experience from the clinical world to the development of new technologies and drugs in the preclinical world. One of the most valuable tools that a technologist can provide in the preclinical imaging and development setting is the assessment of feasibility and practical translation while developing new drugs and technologies. Clinical experience brings a valuable translational view to every project; technologists can provide an applied perspective on the road from bench to bedside.

\section{Good Clinical Practice (GCP)}

GCP is an international ethical and scientific quality standard for designing, conducting, recording, and reporting trials that involve human patients. GCPs are published by the International Conference on Harmonization of Technical Requirements of Registration of Pharmaceuticals for Human Use (ICH) and cover all aspects of clinical trials. A variety of other organizations-in the United States and throughout the world-also oversee how clinical research should be performed to ensure that the rights, safety, and well-being of trial patients are protected and clinical trial data are reliable. Within the 4 overriding ICH sections, efficacy (publication E6) is the most relevant guideline affecting clinical research practices, and all personnel must be familiar with this document. In the United States, the CFR assists clinical researchers, sponsors, and drug manufacturers in complying with GCP. Key regulations include 21 CFR 312, "Requirements for an Investigational New Drug"; 21 CFR 50, "Protection of Human Subjects"; 21 CFR 56, "Institutional Review Boards"; and 21 CFR 46, "HIPPA" (Health Insurance Portability and Accountability Act of 1996). Technologists who follow ICH guidelines for GCP apply a more stringent form of this quality standard during studies (3).

\section{Laboratory GMP}

Drug manufacturing occurs within a controlled environment that promotes strict adherence to procedures consistent with the principles of GMP. The entity responsible for the safety and efficacy of the finished drug product must routinely review critical processes, ensure validation of new procedures before implementation, ensure appropriateness and immediacy of training for all personnel involved with a particular process, and demonstrate that proper change control is in place to prevent drift from accepted procedures over time. Technologists practicing within a GMP environment are responsible for maintenance of competency and adherence to standard operating procedures adopted by a given institution. Examples of such activities include the following:

- Acceptance and use of raw materials that meet quality specifications and are manufactured by a qualified vendor.

- Recognition of controlled environments and associated activities, including proper hygiene and gowning; aseptic manipulations; environmental monitoring; and routine cleaning, disinfecting, and clearance of a given work space.

- Familiarity with microorganisms, including activities aimed at lowering bioburden and speciation.

- Validation of personnel training, analytic equipment, vendors, and the environment.

- Testing of finished drug products, compliance with quality specifications, documentation of good laboratory practices, release of drug products, and addressing drug product complaints and recalls.

The drug product manufacturer is responsible for ensuring adherence to GMP. Examples of regulations for PET drug or radiopharmaceutical manufacturing are 21 CFR part 212, 21 CFR part 211, USP 823, and USP 797. 


\section{Clinical Trials}

Clinical trials of new medical technologies are intended to provide the necessary data for Food and Drug Administration approval for clinical use through the premarket approval process. Additionally, the results of clinical trials provide justification for reimbursement by Centers for Medicare and Medicaid Services and private insurance agencies. Properly designed clinical trials evaluate the safety and efficacy of molecular imaging and therapeutic agents and devices. Selection of appropriate patient populations, clinical indications, and endpoints or outcome measures, as well as adherence to relevant regulations and accepted methodology, are key components of successful clinical trials. Molecular imaging and therapeutic technologies often have unique challenges related to endpoints and outcome measures. Successful completion of clinical trials requires a robust clinical trial design, successful site selection and enrollment, collection of accurate data, and compliance with regulatory requirements.

\section{POTENTIAL PITFALLS AND CHALLENGES}

With every new degree program, there are potential challenges and pitfalls that need to be addressed and considered. Specific to the proposed clinical research program, the need for preceptors and advisors has been identified as an area of challenge. A preceptor or advisor, by definition, is an expert or specialist such as a physician or scientist who gives practical experience and training to a student. Within the clinical research program, it will be important that each student have a strong preceptor to assist throughout the program. The preceptor's role will be to provide additional guidance, as necessary. Within nuclear medicine and molecular imaging, it is becoming increasingly difficult to identify individuals willing to provide their time and expertise to those in training, because of physician shortages in critical subspecialties (4).

\section{EXISTING PROGRAMS}

A need for multimodality, clinically trained, researchbased technologists became evident with the development of emerging technologies at the turn of the last century. The arrival of multimodality imaging devices and innovative treatments created a demand for imaging scientists who have both clinical and research expertise in multimodalities (e.g., PET/CT, SPECT/CT, MR imaging, and sonography) and the delivery of therapeutics. To prepare technologists and therapists for growing opportunities in the development of emerging technologies, a postbaccalaureate, professional master of science degree in molecular imaging and therapeutics was proposed and approved at Saint Louis University in 2011. The program is designed for those completing a baccalaureate degree in the radiologic sciences (e.g., nuclear medicine technology, radiation therapy, MR imaging, sonography, and radiography) who are interested in exploring the opportunities created by the development and implementation of emerging technologies. In this program, technologists acquire the technical skills needed to bridge their knowledge across multiple modalities. A significant component of the program focuses on acquiring the clinical research skills necessary to become an active member of the research team. This component of the graduate curriculum is completed through collaboration with Washington University in St. Louis. Molecular imaging and therapeutics students at Saint Louis University are aligned with primary investigators on research topics identified through the Centers for Clinical Imaging Research in the Mallinckrodt Institute of Radiology at Washington University. Through a series of graduatelevel seminars and courses offered every semester within the program, and completed with the oversight of a mentor, students graduating from the program produce a scholarly project suitable for publication in a peer-reviewed journal or for professional presentation or scholarly equivalent. To ensure that the program meets the expectations and requirements of a graduate-level research program, it is directed by both a physician and a technologist with extensive clinical research knowledge and experience.

Trends in molecular imaging and oncology are moving toward biologic characterization of tumors, prediction of progressions and outcomes, and prediction of treatment. Radiology professionals skilled in critical thinking and collaborative research are in high demand. Trends also include clinical trial technologists and an increasing role in product development and drug development. These graduates have unique skills and can be a valuable asset to the imaging and radiation oncology department. A graduate who is skilled in multiple imaging disciplines, holds a professional graduate degree, and is proficient in collaborative research is rare. The program has established an innovative standard of excellence in education and research for Saint Louis University.

\section{ALTERNATIVE PATHWAYS FOR CLINICAL RESEARCH TECHNOLOGISTS}

\section{Preclinical}

Preclinical imaging specialists possess broad expertise in handling and preparing animal models for preclinical research studies (good laboratory practices) and obtaining imaging data using an array of available preclinical imaging instruments. Knowledge of novel biomarkers, as well as data management and quantification, are key in ensuring successful preclinical imaging studies. Adherence to best practices in radiation safety and good veterinary practices is essential.

\section{Certified Clinical Research Associate (CCRA)- Association of Clinical Research Professionals}

A clinical research associate manages clinical research sites for the sponsor and acts as the study monitor, ensuring the study is conducted according to federal regulations, verifying that data collected by the site are accurate, confirming the eligibility of subjects, checking study drug 
records (if applicable), and filing reports with the sponsor. The sponsor, whose intent is the research of pharmaceuticals, biologics, or devices, may use these individuals either directly or indirectly via contract research organizations or as independent consultants or contractors. The CCRA credential is designated to a clinical research associate who has met eligibility requirements, demonstrated proficiency of specific knowledge and job-related skills, and passed the standardized Association of Clinical Research Professionals clinical research associate certification examination. The CCRA designation formally recognizes that a clinical research associate has met the professional standards set forth by the Association of Clinical Research Professionals.

\section{Certified Clinical Research Coordinator (CCRC)- Association of Clinical Research Professionals}

A clinical research coordinator, regardless of job title, works with study subjects at a clinical research site under the immediate direction of a principal investigator whose research activities are conducted under GCP guidelines. The CCRC credential is designated to a clinical research coordinator who has met eligibility requirements, demonstrated proficiency of specific knowledge and job-related skills, and passed the standardized Association of Clinical Research Professionals clinical research coordinator certification examination. The CCRC designation formally recognizes that a clinical research coordinator has met the professional standards set forth by the Association of Clinical Research Professionals.

\section{Certified Clinical Research Professional (CCRP)- Society of Clinical Research Associates}

CCRPs may have backgrounds in nursing, pharmacy, medical technology, business administration, health record maintenance, statistics, biology, teaching, or other areas. CCRPs work in various settings such as government, industry, or academic medical institutions, including cooperative research groups; academic and private institutions; private offices; pharmaceutical, device, and biotechnology companies; contract research organizations; site management organizations; institutional review boards; independent research and development organizations; or organizations involved in the management of clinical trials. The Society of Clinical Research Associates developed its international certification program to create an internationally accepted standard of knowledge, education, and experience by which clinical research professionals will be recognized as CCRPs in the clinical research community. The standards on which this certification program are based have been set forth by this organization to promote recognition and continuing excellence in the ethical conduct of clinical research and in the protection of human subjects.

\section{Clinical Research Technologist (CRT)}

After completion of the clinical research graduate program, graduates should be able to apply standard scientific practices and techniques such as acquiring standardized imaging while following a study-specific protocol, obtain and accurately document study data, identify and report adverse events appropriately, recognize discrepancies in results, and follow established GCPs and both federal and institutional regulations governing the protection of human subjects in research. They will also be able to contribute to experiments in the laboratory or field to test, prove, or modify theories on the basis of research findings and the experiences of others performing related research, and they may be able to include measurement and recording of observations and collecting, compiling, and processing of data. Individuals may also coordinate operational activities in a research laboratory or a clinical practice, providing support services to researchers, physicians, and technicians; assist in the development and implementation of policies, procedures, and standards to ensure the efficiency, accuracy, and effectiveness of research projects; maintain and perform regular quality control on all PET scanners, dose calibrators, and other imaging equipment; instruct others in protocols, imaging, and instrumentation; and coordinate the ordering, storage, and inventory maintenance of supplies, including any investigational radiopharmaceuticals to be used in a study and instruments essential to the operation of the lab and clinical research department.

\section{CONCLUSION}

Translation of novel biomarkers and technologies associated with molecular imaging requires expertise and skills that are similar to those required for traditional drugs and devices and that are also unique in some respects. Recent successes in molecular imaging technologies for characterization of disease and particularly for treatment stratification and monitoring have expanded interest in the field among industry and academia alike. Future translation of molecular imaging technologies for the reasons above but also as a preferred methodology for drug design and fastthroughput screening of potential therapies and disease targets will lead to many opportunities for molecular imaging specialists. Although the expertise required to successfully translate a molecular imaging technology is great and will undoubtedly require the involvement of a multidisciplinary team, the successful molecular imaging specialist will possess a broad understanding of the entire field.

\section{DISCLOSURE}

No potential conflict of interest relevant to this article was reported.

\section{REFERENCES}

1. National Research Council and Institute of Medicine. Advancing Nuclear Medicine Through Innovation. Washington, DC: National Academies Press; 2007.

2. Gilmore D. Opportunities outside the traditional role of individuals with an educational background in nuclear medicine technology and a bachelor's degree. J Nucl Med Technol. 2013;41:245-246.

3. Research essentials: GCP in clinical research. In: Pathways: the Clinical Trials Network Newsletter. Reston, VA: Society of Nuclear Medicine and Molecular Imaging; June 2013:6.

4. Physician shortages to worsen without increases in residency training. AAMC website. https://www.aamc.org/download/286592/data. Accessed July 30, 2014. 